\title{
Evaluation of the Changes in RR and QT Circadian Rhythms in Bedridden Subjects
}

\author{
Sarah Solbiati ${ }^{1,2}$, Alessia Paglialonga ${ }^{1}$, Lorenzo Costantini ${ }^{3}$, Enrico G Caiani $^{1,2}$ \\ ${ }^{1}$ National Research Council of Italy (CNR), Institute of Electronics, Computer and \\ Telecommunication Engineering (IEIIT), Milan, Italy \\ ${ }^{2}$ Politecnico di Milano, Electronics, Information and Bioengineering Department, Milan, Italy \\ ${ }^{3}$ Azienda Sanitaria Locale Lecce - Cardiovascular Department, Lecce, Italy
}

\begin{abstract}
Prolonged bed rest (BR), often related to hospitalization, chronic diseases and ageing, as well as hospitalized COVid-19 patients, induces reduced functional capacity in multiple body systems and rhythms dysregulation, possibly leading to cardiovascular deconditioning and increased arrhythmogenic risk.

In this study, we analyzed $24 \mathrm{~h}$ Holter ECGs collected from 10 healthy subjects before, during, and after a 10day $B R$, aiming at assessing the decline of the circadian rhythms of $R R$ and ventricular repolarization intervals.

Our results proved that a 10-day BR induced changes in the characteristics of cardiac circadian rhythms, in terms of midline value, oscillation amplitude and acrophase, offering a first insight for the formulation of appropriate countermeasures for improving homeostasis maintenance in hospitalized patients.
\end{abstract}

\section{Introduction}

Prolonged bed rest (BR) is an unnatural state, often related to hospitalization, chronic diseases and ageing, as well as more recently hospitalized COVid-19 patients. The gradual head-wards fluids shift induced by the bedridden position and the lack of physical activity can cause reduced functional capacity in multiple body systems, inducing muscle atrophy, bone density loss, autonomic dysfunction, impaired thermoregulation and immune function, and cardiovascular deconditioning [1]. Also, circadian rhythm disruption has been observed in intensive care unit patients [2]. Circadian rhythms are endogenous oscillations found in almost every physiological process, having a period of approximately 24 hours, which allow the living organism to proactively anticipate and dynamically adapt to the environmental changes. These rhythms are also modulated by external cues, such as light, which is the most important synchronizer, ambient temperature, working and feeding hours, wake/sleep and activity/rest cycles, and the regular alternation between upright and supine position [3].
Prolonged BR, in particular when derived from hospitalization, alters the amplitude and phase of these synchronizers, thus inducing circadian rhythm dysregulation. For this reason, the maintenance of the physiological homeostasis of the bedridden patient is fundamental in improving patient's health condition, or limiting its worsening [3]. In addition, a first mechanistic link between endogenous circadian rhythms and cardiac electrical instability through changes in ventricular depolarization interval duration (QT) has been demonstrated in an animal model [4], thus suggesting the need to further evaluate this aspect also in humans. Accordingly, our aim was to assess progressive changes in RR and QT circadian rhythms during a 10-day horizontal BR, to describe the phenomenon in a controlled environment on healthy volunteers.

\section{Methods}

\subsection{Study population and design}

Ten healthy male volunteers (median $\left(25^{\text {th }} ; 75^{\text {th }}\right.$ percentiles) $21(20.25 ; 24)$ years), were enrolled at the Hospital of Izola (Slovenia), after signed consent, to participate to a 10-day horizontal BR (from August $26^{\text {th }}$ to September $\left.13^{\text {th }}, 2019\right)$. Subjects had no history of cardiovascular disease and were not taking medications of any kind. The study consisted of 2 days of baseline data collection (BDC-2 and BDC-1), 10 days of strict bed rest (BR1 through BR10) and 2 days of recovery $(\mathrm{R}+1$ and $\mathrm{R}+2$ ), starting with $\mathrm{BR}$ conclusion between 9AM and $10 \mathrm{AM}$ at $\mathrm{R}+1$. No countermeasure intervention was applied. Subjects were exposed to natural light through a window in their room: the day started at 7:00AM, and subjects were asked to go to sleep between 10:00PM and 11:00PM. The whole BR protocol, including 21 scientific experiments running in parallel by several research teams, was approved (Clinical Trial.gov database under the number NCT04081467, Official Title: MARS-PRE) by the Institutional Review Board of the Science and Research Centre Koper. 
Table 1. Results of the Cosinor analysis of RR and QTend series expressed as median $\left[25^{\text {th }} ; 75^{\text {th }}\right.$ percentiles $]$.

*: Wilcoxon signed-rank $(\mathrm{p}<0.05)$.

\begin{tabular}{|c|c|c|c|c|c|c|c|}
\hline & & BDC-1 & BR3 & BR5 & BR10 & $\mathrm{R}+1$ & $\mathrm{R}+2$ \\
\hline \multirow[t]{6}{*}{$\mathrm{RR}$} & MESOR & 902 & 991 & 1018 & 998 & 821 & 800 \\
\hline & $(\mathrm{ms})$ & [848;942] & {$[947 ; 1058]^{*}$} & {$[959 ; 1064]^{*}$} & {$[954 ; 1048]^{*}$} & {$[800 ; 865]^{*}$} & {$[744 ; 836]^{*}$} \\
\hline & $\mathrm{OA}$ & 187 & 143 & 164 & 167 & 292 & 198 \\
\hline & $(\mathrm{ms})$ & {$[176 ; 239]$} & {$[127 ; 178]^{*}$} & {$[126 ; 182]^{*}$} & {$[143 ; 198]^{*}$} & {$[242 ; 315]^{*}$} & {$[148 ; 206]$} \\
\hline & $\varphi$ & $3: 53 \mathrm{AM}$ & $3: 13 \mathrm{AM}$ & $2: 20 \mathrm{AM}$ & $2: 31 \mathrm{AM}$ & $2: 45 \mathrm{AM}$ & $3: 14 \mathrm{AM}$ \\
\hline & (h:min) & {$[2: 57 ; 4: 18]$} & {$[2: 46 ; 3: 25]$} & {$[1: 52 ; 2: 29]^{*}$} & {$[2: 04 ; 2: 59]$} & {$[2: 29 ; 3: 01]$} & {$[2: 55 ; 3: 25]$} \\
\hline \multirow[t]{6}{*}{ QTend } & MESOR & 379 & 386 & 394 & 394 & 365 & $727 *$ \\
\hline & (ms) & 37. & {$[38$} & {$[38$} & $\begin{array}{l}{[37} \\
24\end{array}$ & $\begin{array}{l}{[36} \\
49\end{array}$ & 373]* \\
\hline & $(\mathrm{ms})$ & {$[27 ; 40]$} & {$[18 ; 27]^{*}$} & {$[16 ; 26]^{*}$} & {$[18 ; 31]^{*}$} & {$[39 ; 59]^{*}$} & {$[28 ; 51]$} \\
\hline & $\varphi$ & 3:57AM & $3: 13 \mathrm{AM}$ & $2: 14 \mathrm{AM}$ & $2: 28 \mathrm{AM}$ & $2: 52 \mathrm{AM}$ & $3: 11 \mathrm{AM}$ \\
\hline & (h:min) & {$[3: 09 ; 4: 43]$} & {$[2: 47 ; 3: 31]$} & {$[1: 42 ; 2: 26]^{*}$} & {$[1: 55 ; 2: 47]^{*}$} & {$[2: 27 ; 3: 07]^{*}$} & {$[3: 02 ; 3: 34]$} \\
\hline & $\begin{array}{l}\Delta \varphi_{R R-Q T e n d} \\
(\min )\end{array}$ & $\begin{array}{l}-8.4 \\
{[-20.6 ;-1.7]}\end{array}$ & $\begin{array}{l}-0.3 \\
{[-12.3 ; 5.6]}\end{array}$ & $\begin{array}{l}7.8 \\
{[6 ; 8.9]}\end{array}$ & $\begin{array}{l}2.4 \\
{[-6.6 ; 12.9]}\end{array}$ & $\begin{array}{l}4.2 \\
{[0.9 ; 11.4]}\end{array}$ & $\begin{array}{l}-6.9 \\
{[-12.9 ; 0.8]}\end{array}$ \\
\hline
\end{tabular}

Table 2. Distribution of the QTc interval series expressed as median [25th;75th percentiles], and $\Delta(\%)$ with the preceding epoch. *: Wilcoxon signed-rank $(\mathrm{p}<0.05)$.

\begin{tabular}{lllllll}
\hline & BDC-1 & BR3 & BR5 & BR10 & R+1 & R+2 \\
\hline QTc (ms) & $415[398 ; 420]$ & $399[396 ; 407]^{*}$ & $398[389 ; 405]^{*}$ & $395[388 ; 404]^{*}$ & $413[407 ; 427]$ & $415[405 ; 427]$ \\
$\Delta(\%)$ & & $-3.4[-5.5 ;-1.5]^{*}$ & $-0.6[-1.7 ; 0.8]$ & $-0.2[-0.9 ; 0.4]$ & $4.4[2.8 ; 5.5]^{*}$ & $0.2[2.3 ; 1.7]$ \\
\hline
\end{tabular}

\subsection{ECG data acquisition and pre- processing}

For each subject, 12-lead, 24-hour Holter ECGs (1000 $\mathrm{Hz}, \mathrm{H} 12+$, Mortara Instrument Inc.) were acquired at specific epochs (Figure 1): one day before BR (BDC-1), the $3^{\text {rd }}$ (BR3) and $5^{\text {th }}$ (BR5) day of BR, and continuously from the evening of BR9 to subject departure at $R+2$, resulting in three acquisitions, named as BR10 (from the evening of BR9 to the evening of BR10), $\mathrm{R}+1$ (from the evening of BR10 to the evening of $\mathrm{R}+1$ ), $\mathrm{R}+2$ (from the evening of $\mathrm{R}+1$ to the evening of $\mathrm{R}+2$ ).

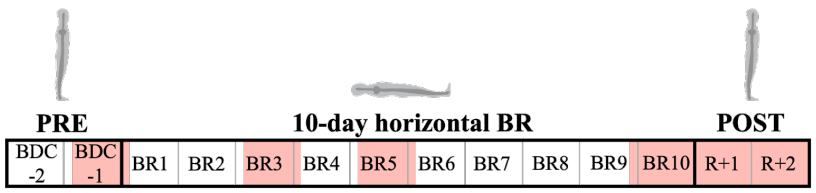

Figure 1. Schematization of bed rest campaign phases. In red, the scheduled epochs of Holter ECG collection.

The research software SuperECG (Mortara Instrument) was used to automatically detect in each Holter recording the fiducial points corresponding to $\mathrm{Q}, \mathrm{R}$ and $\mathrm{T}$ waves, and used to compute beat-to-beat RR and QTend interval series (ms) [5]. The extracted series were pre-processed to exclude outliers or artefacts due to acquisition problems (i.e., electrode detaching, cable interference, others).
Additionally, Bazett's correction [6] was used to compute beat-to-beat QTc interval series. Then, each series was realigned to the time of day start (7:00AM), and median values of consecutive non-overlapping 15minutes segments were computed.

\subsection{Cosinor analysis}

The Cosinor analysis is a widely used method in chronobiology for the evaluation of non-random rhythms in time series, consisting in fitting the original series with a cosine curve [7]. In the analysis of circadian rhythms, the period can be assumed as known (approximately 24h), and the single component regression model is applied. This model is defined with the equation:

$$
\mathrm{Y}(\mathrm{t})=M E S O R+O A \times \cos \left(\frac{2 \pi}{\tau+\varphi}\right)+e(t)
$$

where the MESOR (Midline Statistic of Rhythm) represents a rhythm-adjusted mean, OA is the oscillation amplitude, measuring half variation within a night-day cycle, $\varphi$ is the acrophase, that is the temporal value at which the amplitude of the fitting sinusoid reaches its maximum value, $\tau$ is the period representing the duration of one cycle, and e(t) is the fitting error term.

For each recording, the Cosinor analysis was applied on the series of 15-minute medians of RR and QTend, thus resulting in a value of MESOR, OA, and $\varphi$ for each subject at each epoch, as well as $\Delta \varphi_{R R-Q T e n d}$. 


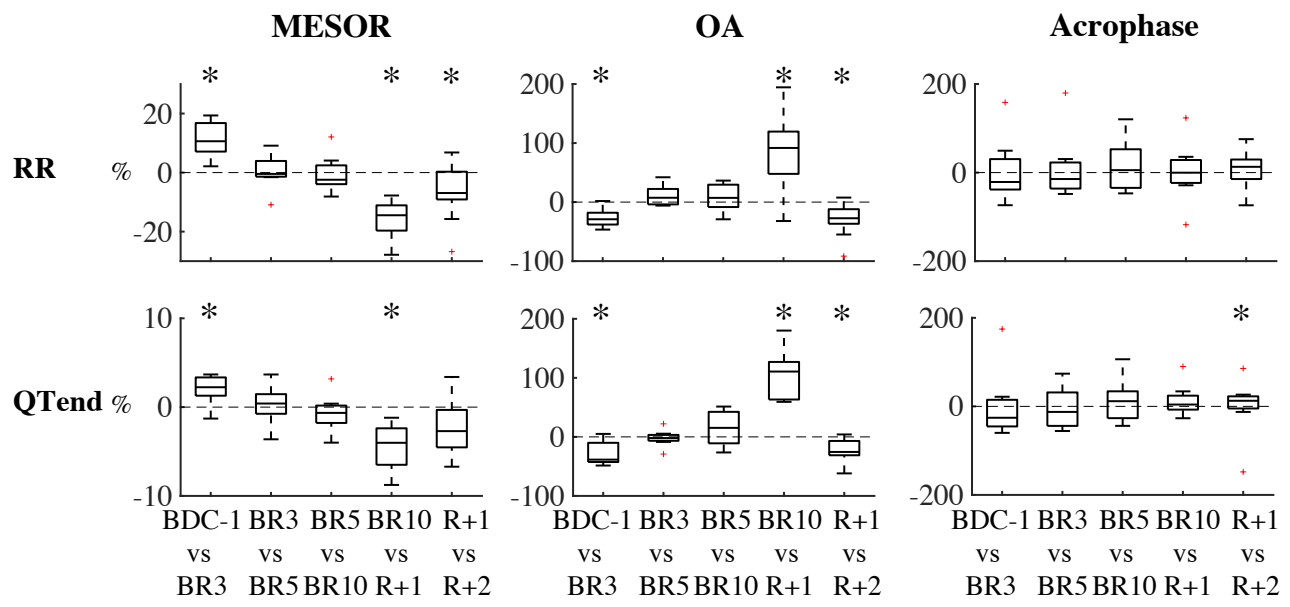

Figure 2. Cumulative distributions of the $\Delta(\%)$ between successive epochs for the MESOR, OA and Acrophase (top to bottom) of RR (left) and QTend (right). Dashed line indicates no change ( $0 \%$ variation). Negative values describe a reduction over time. *: Wilcoxon signed-rank $(\mathrm{p}<0.05)$.

Additionally, the percent difference between successive epochs was computed as:

$\Delta(\%)=100 *\left(\left(\right.\right.$ output $_{i+1}-$ output $\left._{i}\right) \div\left(\right.$ output $\left.\left._{i}\right)\right)$ where $i=1,2, . .5$ are the epochs.

Recordings having less than $60 \%$ of available signal over the 24 hours were excluded from the analysis.

\subsection{Statistical analysis}

To evaluate the effects of BR, the Wilcoxon signedrank test $(\mathrm{p}<0.05)$ was applied to test each epoch against BDC-1, as well as to compare successive epochs.

Additionally, the presence of circadian rhythmicity was assessed with the Zero-Amplitude Test $(\mathrm{p}<0.05)$.

\section{Results}

For technical problems, at BR3 the recordings of two subjects were removed. The results of the Cosinor analysis on RR and QTend are reported in Table 1. As confirmed by the Zero-Amplitude test, the circadianity of $\mathrm{RR}$ and QTend rhythms was maintained in all subjects at all epochs.

During the BR, RR and QTend MESOR were higher than at BDC-1, showing a progressive increase from BR3 to BR5, and remaining prolonged until BR10. Afterwards, their abrupt, progressive decrease was observed at $\mathrm{R}+1$ and $\mathrm{R}+2$. Conversely, the QTc interval progressively decreased up to BR 10 , as reported in Table 2 , then recovering to baseline values at $\mathrm{R}+1$ and $\mathrm{R}+2$. The $\mathrm{OA}$ of $\mathrm{RR}$ and QTend intervals remained decreased during the entire BR. Then, it sharply increased at $\mathrm{R}+1$, recovering to baseline values at $\mathrm{R}+2$. Also, a trend of anticipation of the circadian acrophase in RR and QTend compared to BDC-1 was observed during all $\mathrm{BR}$ and recovery epochs, and particularly for RR at BR5, and
QTend from $\mathrm{BR} 5$ to $\mathrm{R}+1$. However, $\Delta \varphi_{\text {RR-QTend }}$ remained comparable to baseline, although presenting a phase inversion from BR5 to $\mathrm{R}+1$ compared to BDC-1, with $\varphi_{\mathrm{QTend}}$ anticipating $\varphi_{\mathrm{RR}}$.

Abrupt variations between BDC-1 and BR3 were observed in the RR and QTend MESOR and OA, as shown in Figure 2. Also, an opposite and greater variation was visible between BR10 and $\mathrm{R}+1$, while between $\mathrm{R}+1$ and $\mathrm{R}+2$ there was a trend towards recovery. Similarly, the median QTc displayed significant variations, as reported in Table 2, both at the BR start (BDC-1 - BR3) and at BR conclusion (BR10 - R+1).

\section{Discussion}

In this study, the 24h beat-to-beat RR, QTend and QTc variability series extracted from Holter ECG, acquired from 10 healthy subjects undergoing a 10-day horizontal $\mathrm{BR}$, were analyzed.

\subsection{Effects on the 24-hour midline}

During the BR, the midline value of RR increased, well reflecting the reduced daily activity deriving from the BR immobilization. In particular this increase progressed up to BR5, and persisted at BR10. Also, the QTend MESOR mirrored RR variations, thus indicating that the relationship between RR and QT duration was maintained, while the 24-hour QTc interval was found equally shortened during the entire BR duration. No apparent recovery towards the baseline values was detected along the BR, thus showing that the changes are not temporary but persistent during the bedridden position, at least for 10 days. After BR, RR and QTend intervals both shortened compared to baseline and BR epochs, possibly due to the cardiac and autonomic 
deconditioning that prevented post-BR adaptation, with reduced vagal activity at $\mathrm{BR}$ discontinuation [8].

\subsection{Effects on the Oscillation Amplitude}

The 24h RR and QTend OA decreased during the BR, possibly due to the reduction of physical activity and the elimination of the upright/supine cycle, which contribute as synchronizers [3]. The decreased amplitude of the $24 \mathrm{~h}$ variability is a poor prognostic factor for critical illnesses, leading to decreased capacity of adaptation to new stimuli and may underline an autonomic disfunction [3]. Indeed, at $\mathrm{BR}$ conclusion at $\mathrm{R}+1$, the $\mathrm{OA}$ of $\mathrm{RR}$ and $\mathrm{QTend}$ promptly increased, both compared to BDC-1 and to BR10. This abrupt variation may result from the cardiac deconditioning induced by the prolonged $\mathrm{BR}$ that, consequently to the amplitude loss, manifested decreased capacity of adaptation when the normal condition was restored. In mice, deficiency or excess of Krüppel-like factor 15 , with a role in the transcriptional control of the rhythmic genes expression required for generating the transient outward potassium current, has been related to loss of rhythmic QT variation, abnormal repolarization and enhanced susceptibility to ventricular arrhythmias [4]. Besides the known difference in ventricular repolarization between humans and animals [9], fascinating hypothesis of predictive value of 24h QT rhythms in humans for the manifestation of ventricular arrhythmias should be further investigated in patients.

\subsection{Effects on the Acrophase}

The RR and QTend acrophases appeared affected by BR with a pattern of anticipation. However, this trend was constant during the experiment, suggesting a shift in the rhythm (and not free-running). Also, the coupling between $\varphi_{\mathrm{QTend}}$ and $\varphi_{\mathrm{RR}}$ was affected, with $\varphi_{\mathrm{RR}}$ anticipating $\varphi_{\mathrm{QTend}}$, and inverting from BR5 to $\mathrm{R}+1$. An abnormal phase can be caused by altered external synchronizers, (i.e., strength, amplitude and timing), and it is in turn potentially related to increased cardiovascular risk [10].

\section{Conclusions}

The RR and QT circadian rhythms were affected by 10-days bedridden immobilization. These findings provide interesting insights on cardiovascular alterations produced in immobilized or bedridden patients, suggesting that strategies or countermeasures aiming at the maintenance of circadianity in RR and QT should be applied also in clinical scenarios, such as personalized circadian medication [11], in-hospital exercise programs [12], or nutritional interventions [13].

\section{Acknowledgments}

This research was supported by the Italian Space Agency (ASI-MARS-PRE Project, call DC-VUM-2017-006, contract N. 2019-11-U.0).

\section{References}

[1] Convertino, V.A., S.A. Bloomfield, J.E. Greenleaf. An overview of the issues: physiological effects of bed rest and restricted physical activity. Med. Sci. Sports. Exerc. 29(2):187190, 1997.

[2] Korompeli, A., O. Muurlink, N. Kavrochorianou, T. Katsoulas, G. Fildissis, G. Baltopoulos. Circadian disruption of ICU patients: A review of pathways, expression, and interventions. J Crit Care 38:269-277, 2017.

[3] McKenna, H.T., I.K. Reiss, D.S. Martin. The significance of circadian rhythms and dysrhythmias in critical illness. $J$ Intensive Care Soc 18(2):121-129, 2017.

[4] Jeyaraj, D., S.M. Haldar, X. Wan, et al. Circadian rhythms govern cardiac repolarization and arrhythmogenesis. Nature 483(7387):96-99, 2012.

[5] Mortara, D.W. Automated QT measurement and application to detection of moxifloxacin-induced changes. Ann Noninvasive Electrocardiol.14 (Suppl 1):S30-S34, 2009.

[6] Bazett, H.C. An analysis of time relations of electrocardiograms. Heart, 7:353-367, 1920.

[7] Cornelissen, G., Cosinor-based rhythmometry, Theor. Biol. Med. Model. 11:16, 2014.

[8] Crandall, C.G., K.A. Engelke, J.A. Pawelczyk, P.B. Raven, V.A. Convertino. Power spectral and time based analysis of heart rate variability following 15 days head-down bed rest. Aviat. Space Environ. Med. 65(12):1105-1109, 1994.

[9] ICH Harmonised Tripartite Guideline. The NonClinical Evaluation of the Potential for Delayed Ventricular Repolarization (QT Interval Prolongation) by Human Pharmaceuticals. S7B Step 4 version, 2005.

[10] Virkkunen, H., M. Härmä, T. Kauppinen, L. Tenkanen. Shift work, occupational noise and physical workload with ensuing development of blood pressure and their joint effect on the risk of coronary heart disease. Scand. J. Work Environ. Health 33(6):425-434, 2007

[11] Dallmann, R., S.A. Brown, F. Gachon Chronopharmacology: new insights and therapeutic implications. Annu. Rev. Pharmacol. Toxicol. 54:339-361, 2014. [12] McCullagh, R., E. O'Connell, S. O’Meara, D. Dahly, E. O'Reilly, K. O'Connor, N.F. Horgan, S. Timmons. Augmented exercise in hospital improves physical performance and reduces negative post hospitalization events: a randomized controlled trial. BMC Geriatr. 20:46, 2020.

[13] Solbiati, S., F. Landreani, M. Turcato, L. Costantini, P. Vaida, E.G. Caiani. Analysis of changes in cardiac circadian rhythms of RR and QT induced by a 60-day head-down bed rest with and without nutritional countermeasure. Eur. J. Appl. Physiol. 120(7):1699-1710, 2020.

Address for correspondence:

Prof. Enrico G. Caiani

Electronic, Information and Bioengineering Dpt.

Politecnico di Milano, P.zza L. da Vinci 32, 20133 Milano

Email: enrico.caiani@polimi.it 